\title{
Resistance and utilisation pattern of antibacterial agents in outpatient settings in two Teaching Hospitals in Colombo
}

\author{
G P S G Senadheera' ${ }^{1,3}$, S Sri Ranganathan ${ }^{3}$, G Patabendige ${ }^{4}$, G H Fernando ${ }^{2}$, D Gamage ${ }^{4}$, \\ R M W Maneke ${ }^{5}$, B M R Fernandopulle ${ }^{6}$
}

(Index words: antibacterial resistance, community, Escherichia coli, surveillance)

\begin{abstract}
Objectives Antibacterial resistance (ABR) is a public threat. Sri Lanka is a country with limited surveillance of $\mathrm{ABR}$ in the community. The WHO methodology was adapted to identify ABR in outpatient settings (nonhospitalised patients) and its link to consumption of antibiotics.

Methods It was a cross-sectional descriptive community based study to collect ABR data from Out Patient Department (OPD) of two leading Teaching Hospitals in Colombo district. The indicator organism Escherichia coli (E. coli) was obtained from the urine specimens of patients who were suspected to have urinary tract infections. Antibiotic susceptibility testing was performed for commonly used oral antibiotics using disc diffusion method. The antibiotic consumption aggregate data were collected from the OPD pharmacies of the said hospitals and expressed as Defined Daily Doses (DDD) per 1000 inhabitants per 1000 day.

Results Of the 2183 urine samples, pathogenic E. coli was isolated in $9.3 \%(204)$, and $8 \%(n=16)$ of them were Extended Spectrum Beta Lactamase (ESBL) producers. E.coli was most resistant to ampicillin (85\%), followed by nalidixic acid (58.5\%), trimethoprim/sulphamethoxazole (47.1\%), ciprofloxacin (46.2\%), norfloxacin (43.7\%) amoxicillin /clavulanic acid (36.3\%) and nitrofurantoin (15\%). Multi-drug resistance was seen in 44\%. Amoxicillin was the most frequently consumed antibacterial agent (2.65 DDD per 1000 inhabitants per day).

Conclusions There is an alarmingly high antibiotic resistance in the non-hospitalised patients indicating high prevalence of $E$. coli resistance in the community.
\end{abstract}

Ceylon Medical Journal 2016; 61: 113-117

DOI: http://doi.org/10.4038/cmj.v61i3.8346

\section{Introduction}

Antibacterial resistance (ABR) is a global health problem. Developed countries have implemented different evidence based strategies to contain the problem such as surveillance of ABR, monitoring antibacterial use, national antibacterial policies including rational use of antibacterials [1]. However, in developing countries where the problem is greater [2], financial resources are spent in procuring second, third and even fourth line antimicrobials, although it is well known that 'the more we use antimicrobials, the more we lose them'. This cycle can be effectively stopped in developing countries only if they implement urgent and long lasting measures to contain the emerging problem of ABR.

The World Health Organization (WHO) recommends key interventions for containment of ABR which can be adapted to suit the needs and resources of individual countries [3]. One such key intervention is establishment of effective and epidemiologically sound surveillance programmes for ABR and antimicrobial use targeting different settings such as hospitals, community and intensive care units [4]. Many developed countries have such surveillance programmes built into their healthcare structure, whereas developing countries lack motivation, policy framework and resources to establish such programmes [5]. The Sri Lanka College of Microbiologists has conducted two hospital based ABR surveillances studies, but up to now, community based surveillances were not conducted [6]. In addition, ABR data have not been compared with antimicrobial use, either in hospital or community settings.

Studies from many countries have reported that surveillance ABR data in a community had been effective and instrumental in the containment of ABR as they have

Departments of ${ }^{1}$ Allied Health Sciences and ${ }^{2}$ Pharmacology, Faculty of Medical Sciences, University of Sri Jayewardenepura, ${ }^{3}$ Department of Pharmacology, Faculty of Medicine, University of Colombo, ${ }^{4}$ Department of Microbiology, National Hospital of Sri Lanka, Colombo, ${ }^{5}$ Department of Microbiology, Colombo South Teaching Hospital, ${ }^{6}$ Department of Paraclinical, General Sir John Kotelawala Defence University, Ratmalana, Sri Lanka.

Correspondence: GPSGS, <savini@sjp.ac.lk>. Received 26 January and revised version accepted 31 May 2016.

This is an open-access article distributed under the terms of the Creative Commons Attribution License, which permits unrestricted use, distribution, and reproduction in any medium, provided the original author and source are credited. 
the potential to help countries in prioritizing interventions, determining effectiveness of interventions and in developing antibiotics guidelines [7].

To support such surveillance programmes in resource constrained settings, WHO has developed a methodology for community based surveillances studies [8]. It recommends to collect data from hospital outdoor patients for community based surveillances. Such methodology has been piloted in India and South Africa, and researchers have shown methodological issues relevant to their respective countries [8]. In this study, we have adapted the WHO methodology to overcome identified methodological issues and attempted to identify ABR pattern in outpatient settings reflecting $A B R$ pattern in the community and link it to antibiotic consumption.

\section{Methods}

Ours was a cross-sectional descriptive study where data were collected from two teaching hospitals in Colombo district over a one-year period from June 2013 to May 2014. These two teaching hospitals serve a large number of patients and have a sizeable laboratory turnover. Urine from patients with urinary tract infection (UTI) have been identified as the most suitable specimen to describe community based ABR trends [9]. This is mainly due to two reasons; (i) UTI is a common bacterial infection seen in the community and (ii) specimen (urine) is easily obtainable for microbiological investigations. According to the WHO methodology, Escherichia coli was identified as the best indicator organism for the purpose of this study [6,9].

Patients who attended the outpatient departments (OPD) or medical clinics of these hospitals with symptoms suggestive of UTI, and the doctor had requested urine culture and antibiotic susceptibility test (AST) were selected for the study. The WHO methodology recommends to include only outpatients for community based surveillance. Those who were hospitalised during the past 48 hours (as it may not be a community acquired UTI), patients who reported taking antibiotics within 5 days (as it will interfere with the urine investigations results), and patients residing outside Colombo (as the study was confined to Colombo district) were excluded.

As per the laboratory procedures for outpatients, urine samples were either brought from home in a urine culture bottle collected from the laboratory or obtained from patients in urine culture bottles when they visited the laboratory. Principal investigator or a data collector was stationed at these microbiology laboratories in the morning during the study period when urine samples were accepted for investigations. A pre-tested, structured, interviewer administered proforma was used to collect the required data such as recent use of antibiotics, recent hospitalisation and demographic details.

Samples were inoculated on chromogenic urinary tract agar medium plates (Oxoid Ltd, UK) using the standard techniques followed in the respective laboratories and incubated in air at $35-37^{\circ} \mathrm{C}$ for $18-24$ hours. Growth of pink colonies was presumed to be $E$ coli and later identified using standard techniques.

In adults a colony count of $>10^{5}$ colony forming units (CFU) / ml of E coli in pure growth was considered a significant pathogenic Escherichia coli growth. In children a colony count $>$ or $=10^{5} \mathrm{CFU} / \mathrm{ml}$ and $10^{4}-10^{5}$ $\mathrm{CFU} / \mathrm{ml}$ of $E$ coli in pure growth was considered a significant pathogenic Escherichia coli growth. The urine samples which showed significant pathogenic E coli growth were selected for this study. Antibiotic susceptibility testing (AST) was performed for significant isolates of $E$ coli using the disc diffusion test method according to Clinical Laboratory Standard Institute (CLSI) 2011 guidelines. Screening and phenotypic confirmatory test for Extended Spectrum Beta Lactamase (ESBL) positive $E$ coli was also done as per the same guidelines.

Isolated $E$ coli was tested for its susceptibility for following antibiotics in children (under the age of 12 years) ampicillin, cephalothin, cefuroxime, nalidixic acid, amoxicillin-clavulanic acid, nitrofurantoin, and trimethoprim / sulphamethoxazole. In adults cephalothin was replaced with norfloxacin and ciprofloxacin. For each antimicrobial agent, the isolates were categorised as susceptible, intermediate or resistant based on the CLSI standards. The latter two categories were grouped together and considered as resistant when data were analysed. Multidrug resistance (MDR) was defined as resistance to at least one member from three different classes of antibacterial agents included in the treatment of UTI [10].

For community based surveillance of antimicrobial use in developing countries, the WHO methodology recommends obtaining antimicrobial supply or prescription data from the same hospitals as collecting data on actual use of antimicrobials by the patients in the target community is logistically impossible. This method has been successful in many studies [8, 9]. In other words, it is assumed that the antimicrobial utilization data from the study hospitals represent the antimicrobial use in respective communities. Data on antimicrobial use can be either prescription based data obtained from individual patients visiting the OPD or aggregate data obtained from hospital OPD pharmacy of the hospital. We selected the aggregate data for the purpose of this study.

Details (name, dosage form, dose, strength and amount) of antimicrobials dispensed over a period of one year (October 2012 - September 2013) during the study period were extracted from the records maintained in the OPD pharmacies of both hospitals. As per the national medicines supply system, the antimicrobials supplied to OPD pharmacies are restricted to amoxicillin, nitrofurantoin, nalidixic acid and trimethoprim/sulphamethoxazole. Microsoft excel programme was used for data entry and annual supply of each antimicrobial agent was converted to Defined Daily Doses (DDD). The DDD is the 
universally accepted quantification unit for drug utilisation studies. It is defined as 'the assumed average maintenance dose per day for a drug used for its main indication in adults' [11].

We calculated the annual supply of an antimicrobial agent in terms of DDD as follows:

$\operatorname{DDD}($ Annual $)=$ Strength of the antimicrobial agent $\times$ amount of supply

DDD for the antimicrobial agent

WHO Collaborating Centre for Drug Statistics Methodology in Oslo provides DDD for the antimicrobial agent [11]. As per standard drug utilisation terminology, we have expressed the consumption as DDD/1000 population /day [12]. The calculation is shown below:

$=$ DDDs dispensed at the OPD pharmacies of the two hospitals during the study period $\times 1000$

Number of patients attended the OPDs of the two hospitals during the study period $\times 365$

Descriptive statistics were used to analyse data. Approval was obtained from the Ethics Review Committee of Faculty of Medicine, University of Colombo (EC-11121). Administrative approval was obtained from all relevant authorities.

\section{Results}

During the study period of one year, 2183 urine samples from out patients were received in these two laboratories. Significant pathogenic $E$ coli growth was isolated in 204 (9.34\%). Of the isolated E coli, 8\% (16/204) were ESBL secreting bacteria. Mean age of the patients was 50 (range, 1-83) year with a male to female ratio of 1:4. Of the 204 urine samples positive for $E$ coli, females between 35 to 64 years accounted for 37\% (Table 1).

Resistance to ampicillin, nalidixic acid, ciprofloxacin and norfloxacin was observed in 85\%, 58.5\%, 46.2\% and $43.7 \%$ of isolates respectively (Table 2 ). Of the 192 E.coli isolates tested for the three quinolones (nalidixic acid, norfloxacin, ciprofloxacin), about one-third (38\%) were resistant to all three quinolones and another one-third (36.6\%) was susceptible to all three quinolones. Of the 196 E. coli isolates tested for ampicillin (considered as equivalent to amoxicillin in AST procedures) and coamoxiclav, 43\% were resistant to both drugs. Resistance only to ampicillin and not to co-amoxiclav was observed in $37 \%$ of the remaining isolates. Forty four of the 204 isolates were mutidrug resistant Escherichia coli. Amoxicillin is the leading antibiotic issued in the OPD pharmacies (Table 3). The graphical relationship of antibiotic use and resistance percentages in the two teaching hospitals is depicted in Figure 1.

Table 1. Distribution and frequency of $E$. coli pathogenic isolates

\begin{tabular}{ccccccc}
\hline Age group & $F$ & $\begin{array}{c}\text { \% (from total } \\
\text { isolates) }\end{array}$ & $M$ & $\begin{array}{c}\text { \% (from total } \\
\text { isolates) }\end{array}$ & Grand total & $\%$ \\
\hline $0-12$ & 8 & 3.9 & 9 & 4.4 & 17 & 8.3 \\
$13-34$ & 22 & 10.8 & 3 & 1.5 & 25 & 12.2 \\
$35-64$ & 76 & 37.2 & 20 & 9.8 & 96 & 47.1 \\
$>65$ & 44 & 21.6 & 22 & 10.8 & 66 & 32.4 \\
Total & 150 & 73.5 & 54 & 26.5 & 204 & 100 \\
\hline
\end{tabular}

Table 2. Antibiotic resistance pattern of Escherichia coli isolated from the urine samples of patients attending the OPDs of two Teaching hospitals in Colombo district

\begin{tabular}{lcc}
\hline Antibiotic & Number tested & Number of resistant isolates (\%) \\
\hline Ampicillin & 199 & $169(85)$ \\
Nalidixic acid & 200 & $117(58.5)$ \\
Trimethoprim /sulphamethoxazole & 204 & $96(47.1)$ \\
Ciprofloxacin & 199 & $92(46.2)$ \\
Norfloxacin & 199 & $87(43.7)$ \\
Amoxicillin /clavulanic acid & 201 & $73(36.3)$ \\
Cefuroxime & 189 & $65(34.4)$ \\
Cepholothin* & 8 & $2(25)$ \\
Nitrofurantoin & 202 & $30(15)$ \\
\hline
\end{tabular}

*only for children as one hospital has paediatric patients 
Table 3. ABM issued from OPDs of two teaching hospitals during the period 2012 August to 2013 September 2013

\begin{tabular}{lcc}
\hline Antibiotic & DDDs & $\begin{array}{c}\text { DDD per 1000 } \\
\text { inhabitant day }\end{array}$ \\
\hline Amoxicillin & 1131397.7 & 2.65 \\
Nalidixic acid & 16261.5 & 0.04 \\
Nitrofurantoin & 29728.8 & 0.07 \\
Trimethoprim/sulphamethoxazole & 5421 & 0.01 \\
Total & 1230710.7 & 2.88 \\
\hline
\end{tabular}

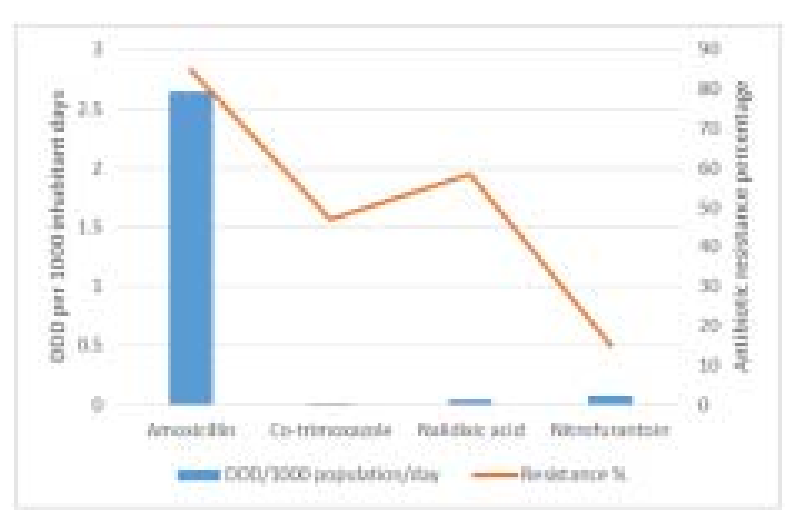

Figure 1. Antimicrobial resistance vs. antimicrobial use in two teaching hospitals.

\section{Discussion}

Our study indicates that the resistance of $E$ coli to commonly used antibiotics for treatment of UTI exceeds the threshold of $20 \%$. Resistance to ampicillin is as high as $85 \%$. In AST, resistance to ampicillin is used as an indicator for resistance to amoxicillin. An Indian study which used the same methodology reported resistance to ampicillin as $84.6 \%$ [9]. Even global data indicate that E.coli is less sensitive to ampicillin [9, 13-16]. However, the rate is about $50 \%$ in most of the developed countries. $[13,16,17]$. Ampicillin/amoxicillin is a safe and relatively cheap antibacterial agent available for treatment of uncomplicated infections in the community. However, developing countries such as India and Sri Lanka seem to have failed to preserve this antibiotic, whereas the developed countries who could afford more expensive second line antibacterial agents have fared better. In the OPD only certain antibiotics are available such as amoxicillin, cloxacillin, erythromycin, nalidixic acid, nitrofurantoin, and trimethoprim/sulphamethoxazole. Others are restricted for use in clinics. Our results also indicate that resistance to quinolones has reached approximetly 50\% (ciprofloxacin 46.2\%, norfloxacin $43.7 \%$, nalidixic acid 58.5\%). Global data also indicate that resistance to quinolones is on the increase. A Canadian study reported that resistance to ciprofloxacin has increased from 1-1.8\% in 2000-2001 to 7\% in 2004, and data from London indicated that it has increased from $1.9 \%$ in 2005 to $7.4 \%$ in 2008 [16,17]. The high level of resistance in our study is alarming when compared with global data. Although use of ciprofloxacin is low in the OPD of government hospitals of Sri Lanka, a previous study done showed it to be high in the private community setting [18].

In our data, utilisation of amoxicillin (2.65/1000 population/day) was much higher than that of nalidixic acid (0.04/ 1000 population/ day) and cotrimoxazole (0.01/ 1000 population/ day), but the difference was not reflected in the percentage of resistance (ampicillin 85\%, nalidixic acid 58.5\%, cotrimoxazole $47 \%$ ). In government hospital OPDs, amoxicillin is prescribed for many non-UTI infections such as respiratory infection, sinusitis and dental infections. Prescribing nalidixic acid or cotrimoxazole is mainly limited to UTI and dysentery. This may account for the disproportionately high use of amoxicillin reflected in our data. E. coli was found to be least resistant to nitrofurantoin similar to studies from Canada, London and US $[13,16,17]$. Hence prescribers should be encouraged to use nitrofurantoin as first line drug in empirical treatment of UTI.

We have no data on utilisation of ciprofloxacin as it is not supplied to OPD pharmacies in the government hospitals. However, possibility of prescriptions for ciprofloxacin given by doctors to be purchased from outside cannot be ruled out. A significant association has been reported between amoxicillin use and fluoroquinolone resistance [19]. The high utilisation of amoxicillin would have contributed to ciprofloxacin resistance observed in our study.

The rate of ESBL producing E. coli in our study is similar to that among out patients in a tertiary care setting [20]. Previously ESBL producing E.coli was shown to be a problem in the hospital settings only, however the problem has been reported to be extending to community settings 
as well [21]. Results of our study also show that it has become a problem even in the community.

In conclusion, the extent of antimicrobial resistance shown by $E$ coli is high and it reflects the AMR pattern in the community in Colombo District. The WHO methodology of community surveillance AMR was adaptable to generate data from a resource limited setting such as ours.

\section{Funding}

Higher Education for the Twentieth Century Project - HRD /Ministry of Higher Education, Sri Lanka.

\section{Acknowledgements}

Authors thank Dr. Shirani Chandrasiri, Consultant Microbiologist, Colombo South Teaching Hospital, all staff of the Microbiology Laboratory of the Colombo South Teaching Hospital and National Hospital of Sri Lanka, and Dr. Philomina Chandrasiri, Former Consultant Microbiologist, National Hospital of Sri Lanka.

\section{Conflicts of interests}

There are no conflicts of interest.

\section{References}

1. Harbarth S, Samore MH. Antimicrobial resistance determinants and future control. Emerg Infect Dis 2005; 11: 794-801.

2. Laxminarayan R, Duse A, Wattal C, Zaidi AK, Wertheim HF, Sumpradit N, et al. Antibiotic resistance - the need for global solutions. The Lancet infectious dis 2013; 13: 1057-98.

3. Leung E, Weil DE, Raviglione M, Nakatani H. The WHO policy package to combat antimicrobial resistance. Bull World Health Organ 2011; 89: 390-2.

4. Archibald L, Phillips L, Monnet D, McGowan JE, Tenover F, Gaynes R. Antimicrobial resistance in isolates from inpatients and outpatients in the United States: increasing importance of the intensive care unit. Clin Infect Dis 1997; 24: 211-5.

5. Ironmonger D, Edeghere O, Bains A, Loy R, Woodford N, Hawkey PM. Surveillance of antibiotic susceptibility of urinary tract pathogens for a population of 5.6 million over 4 years. J Antimicrob Chemother 2015; dkv043.

6. Chandrasiri P, Elwitigala J, Nanayakkara G, Chandrasiri S, Patabendige G, Karunanayaka L, et al. A multi centre laboratory study of Gram negative bacterial blood stream infections in Sri Lanka. Ceylon Med J 2013; 58: 56-61.

7. Mölstad S, Erntell M, Hanberger H, Melander E, Norman C, Skoog G, et al. Sustained reduction of antibiotic use and low bacterial resistance: 10-year follow-up of the Swedish Strama programme. Lancet Infect Dis 2008; 8: 125-32.

8. Holloway K, Mathai E, Sorensen T, Gray A. Communitybased surveillance of antimicrobial use and resistance in resource-constrained settings. Geneva: World Health Organization, 2009.

9. Holloway K, Mathai E, Gray A. Surveillance of antimicrobial resistance in resource-constrained settings experience from five pilot projects. Trop Med Int Health 2011; 16: 368-74.

10. Magiorakos AP, Srinivasan A, Carey R, Carmeli Y, Falagas $\mathrm{M}$, Giske C, et al. Multidrug resistant, extensively drug resistant and pandrug - resistant bacteria: an international expert proposal for interim standard definitions for acquired resistance. Clin Microbiol Infect 2012; 18: 268-81.

11. ATC/DDD Index. Oslo, World Health Organization Collaborating Centre for Drug Statistics Methodology. http://www.whocc.no (accessed on Jan 10, 2016).

12. World Health Organization. Introduction to drug utilization research. World Health Organization, 2003.

13. Sanchez GV, Master RN, Karlowsky JA, Bordon JM. In vitro antimicrobial resistance of urinary E. coli among US outpatients from 2000 to 2010. Antimicrob Agents Chemother 2012: AAC. 06060-11.

14. Cullen IM, Manecksha RP, McCullagh E, Ahmad S, O’Kelly F, Flynn RJ, et al, The changing pattern of antimicrobial resistance within 42033 Escherichia coli isolates from nosocomial, community and urology patient specific urinary tract infections, Dublin, 1999-2009. BJU international 2012; 109: 1198-206.

15. Al Benwan K, Al Sweih N, Rotimi VO. Etiology and antibiotic susceptibility patterns of community - and hospital-acquired urinary tract infections in a general hospital in Kuwait. Med Princ Pract 2010; 19: 440-6.

16. Bean DC, Krahe D, Wareham DW. Antimicrobial resistance in community and nosocomial Escherichia coli urinary tract isolates, London 2005-2006. Ann Clin Microbiol Antimicrob 2008; 7: 13.

17. Alos JI, Serrano MG, Gomez-Garces JL, Perianes J. Antibiotic resistance of Escherichia coli from communityacquired urinary tract infections in relation to demographic and clinical data. Clin Microbiol Infect 2005; 11: 199-203.

18. Senadheera GPSG, Sri Ranganathan S, Fernando GH, Fernandopulle BMR. Newer broad spectrum antibitic use in the Out Patient Departments (OPD) of three major private hospitals in the capital city of Sri Lankain EuroDurg 2014. University Medical Centr, Groningen, The Netherlands 2014.

19. Bergman M, Nyberg ST, Huovinen P, Paakkari P, Hakanen AJ. Association between antimicrobial consumption and resistance in Escherichia coli. Antimicrobial Agents and Chemotherapy 2009; 53: 912-7.

20. Dissanayake DMBT, Fernando SSNF, Chandrasiri NS. The distribution and characteristics of Extended-Spectrum $\beta$ Lactamase (ESBL) producing Escherichia coli and Klebsiella species among urinary isolates in a tertiary care hospital. Sri Lanka Journal of Infectious Diseases 2012; 2: 30-6.

21. Ena J, Arjona F, Martínez-Peinado C, del mar LópezPerezagua M, Amador C. Epidemiology of urinary tract infections caused by extended-spectrum beta-lactamaseproducing Escherichia coli. Urology 2006; 68: 1169-74. 